\title{
Percepção de graduandos e cirurgiões-dentistas acerca dos resíduos gerados na clínica odontológica e seu impacto ambiental: uma Revisão Integrativa
}

\author{
Perception of undergraduates and dentists about the waste generated in the dental clinic and its \\ environmental impact: an Integrative Review \\ Percepción de universitarios y odontólogos sobre los residuos generados en la clínica dental y su \\ impacto ambiental: una Revisión Integradora
}

Recebido: 01/12/2021 | Revisado: 08/11/2021 | Aceito: 09/12/2021 | Publicado: 16/12/2021
Hanna Marrie Dantas Raposo da Câmara ORCID: https://orcid.org/0000-0002-5947-1435 Universidade do Estado do Rio Grande do Norte, Brasil E-mail: hannamarrie@alu.uern.br Glenda Vieira de Sousa ORCID: https://orcid.org/0000-0003-2366-9623 Universidade do Estado do Rio Grande do Norte, Brasil E-mail: glendavieira@alu.uern.br
Samara Carollyne Mafra Soares ORCID: https://orcid.org/0000-0002-2346-9528 Universidade do Estado do Rio Grande do Norte, Brasil E-mail: samaramafra@uern.br

\begin{abstract}
Resumo
Objetivo: Verificar o conhecimento de graduandos de Odontologia e cirurgiões-dentistas acerca dos resíduos gerados após o atendimento clínico e seu impacto ambiental. Metodologia: Trata-se de uma revisão integrativa, cuja estratégia de busca se baseou na utilização das palavras e descritores e selecionou artigos publicados na língua portuguesa, inglesa e espanhola, disponíveis na integra e na forma online nas bases de dados PubMed e SciELO, além de MEDLINE, LILACS e BBO via BVS e compreendidos no ano de 2008 a 2019, coletados nos meses de fevereiro a março de 2021. Dentre os 49 títulos e após aplicação de filtros, leitura de títulos e resumos e remoção de duplicatas, totalizou 8 estudos para compor esta revisão. Resultados: A maioria dos estudos apontaram falhas no conhecimento sobre os RSS, seja na amostra dos graduandos ou cirurgiões dentistas, e quando o conhecimento dos mesmos era favorável, poucas atitudes foram tomadas acerca dessa questão. Conclusão: A educação ambiental na prática clínica ainda não é uma realidade vivenciada cotidianamente nos cursos de Odontologia, tampouco praticada por cirurgiões-dentistas. Assim, evidenciase a necessidade de ações intervencionistas em saúde no âmbito acadêmico e profissional, visando a mudança comportamental e favorecendo a sustentabilidade ambiental.

Palavras-chave: Resíduos odontológicos; Gerenciamento de resíduos; Contenção de Riscos biológicos; Resíduos de serviços de saúde.

Abstract

Objective: The objective of this study was to verify the knowledge of undergraduates of Dentistry and dentists about the residues generated after clinical care and its environmental impact. Methodology: This is an integrative review, whose search strategy was based on the use of words and descriptors Dentistry and selected articles published in the Portuguese language, English and Spanish, available in full and online in databases PubMed and SciELO, besides, MEDLINE, LILACS and BBO track BVS and comprised in the year 2008 to 2019, collected in the months of February to March 2021. Among the 49 titles and after applying filters, reading titles and abstracts and removing duplicates, totaled 8 studies to compose this review. Results: The most studies pointed out gaps in knowledge about RSS, whether among undergraduate students or dentists, and when their knowledge was favorable, few attitudes were taken on this issue. Conclusion: The environmental education in clinical practice is not yet a reality experienced daily in dentistry universities, nor practiced by dentists. Thus, the need for interventionist actions in health in the academic and professional scope is evident, aiming at behavioral change and favoring environmental sustainability.
\end{abstract}

Keywords: Dental waste; Waste management; Containment of biohazards; Medical waste.

\section{Resumen}

Objetivo: Verificar el conocimiento de graduandos de Odontología y cirujanos-dentistas acerca de los residuos generados tras de la atención clínica y su impacto ambiental. Metodología: Se trata de una revisión integrativa, cuya estrategia de búsqueda se basó en la utilización de palabras y descriptores y seleccionó artículos publicados en la lengua 
portuguesa, inglesa y española, disponibles en la integra y en la forma online en las bases de datos PubMed y SciELO, además de MEDLINE, LILACS y BBO vía BVS y comprendidos en el año 2008 a 2019, recogidos en los meses de febrero a marzo de 2021. Entre los 49 títulos y después de aplicar filtros, leer títulos y resúmenes y eliminar duplicados, totalizó 8 estudios para componer esta revisión. Resultados: La mayoría de los estudios señalaron brechas en el conocimiento sobre RS, sea en la muestra de estudiantes de pregrado o dentistas y cuando su conocimiento fue favorable, se tomaron pocas actitudes sobre este tema. Conclusión: La educación ambiental aún no es una realidad vivida a diario en los cursos de Odontología, ni practicada por los odontólogos. Así, se evidencia la necesidad de acciones intervencionistas en salud en el ámbito académico y profesional, orientadas al cambio de comportamiento y favoreciendo la sostenibilidad ambiental.

Palabras clave: Residuos dentales; Gestión de residuos; Contención de peligros biológicos; Residuos de los servicios sanitarios.

\section{Introdução}

A atuação na Odontologia está constantemente exposta ao risco de contaminação e a mesma não se restringe à equipe profissional, mas também engloba pacientes e por isso requer cuidados especiais. Assim, medidas de proteção são fundamentais, como, uso de equipamentos de proteção individual, preparo do ambiente e do instrumental, desinfecção e esterilização, e descarte apropriado de resíduos (Pinto, Motta \& Raptopoulos, 2015). O descarte incorreto de resíduos gerados na atividade clínica odontológica se torna, progressivamente, o motivo de preocupação, pois, através desse descarte inadequado, o meio ambiente é contaminado (Molina et al., 2017) (Kemparaj, Panchmal \& Kadalur, 2018).

Os resíduos de serviços de saúde (RSS) são, segundo a Associação Brasileira de Normas e Técnicas (ABNT), todo tipo de lixo produzido em instituições sanitárias e seu manejo é uma forma de gerenciá-los (Associação Brasileira de Normas e Técnicas [ABNT], 1993). Nesse sentido, esses resíduos possuem características tóxicas e possivelmente patogênicas e, com isso constituem um problema para sociedade e meio ambiente (Gomes \& Esteves, 2012).

Nos consultórios odontológicos, são produzidos resíduos: Biológicos, como luvas, máscaras, gaze, algodão ou qualquer material que tenha sido contaminado por fluidos corporais; Químicos, como anestésicos, efluentes de processadores de imagem (reveladores e fixadores), saneantes e desinfetantes, resíduos de amálgama e radiografias odontológicas; Perfurocortantes, como bisturis, agulhas, ampolas de vidro, brocas, limas endodônticas e pontas diamantadas; e resíduos comuns, dentre os quais são encontrados diversos materiais recicláveis, capazes de retornar à cadeia produtiva (Agência Nacional de Vigilância Sanitária [ANVISA], 2006). E devido a esses produtos da clínica, é necessário que o enfrentamento dessa problemática seja realizado pelos profissionais, os quais devem desenvolver competências e habilidades desde a época de graduação, a fim de exigir dos mesmos um posicionamento consciente e colaborativo para a busca de soluções (Gessner, Piosiadlo, Fonseca \& Larocca, 2013).

Diante do exposto, percebe-se que os resíduos de saúde pública são questões atuais que fazem parte do cotidiano da prática clínica, assim, um caminho para solucionar a questão dos RSS é o exercício do bom senso, aliado à educação, treinamento de profissionais da saúde e esclarecimento da população (Diaz et al., 2013).

A partir disso, é fundamental que os discentes possuam o conhecimento prévio sobre os RSS e o Plano de Gerenciamento de Resíduos de Serviços de Saúde (PGRSS), que tem como objetivo minimizar a produção e proporcionar aos resíduos gerados um encaminhamento seguro, e que seja trabalhado nas universidades o assunto, para que a tomada de medidas no contexto da biossegurança, aliado a economia de recursos, preservação ambiental, ética e responsabilidade garantam mais qualidade de vida para as gerações seguintes, pois, para transformar essa realidade, é necessário um trabalho integrado com todos os seguimentos da sociedade, com vistas à implementação de programas de educação ambiental (Garcia \& Zanetti-Ramos, 2004). Nesse contexto, o desenvolvimento sustentável na Odontologia é uma temática que deve ser estimulada, com o objetivo de amenizar a poluição e não comprometer as gerações futuras (Turma do Bem, 2009).

O objetivo deste estudo é verificar o grau de conhecimento de graduandos de Odontologia e cirurgiões-dentistas acerca dos resíduos gerados após o atendimento clínico e seu impacto ambiental. 


\section{Metodologia}

Trata-se de um estudo de revisão integrativa, que utiliza métodos de identificação, análise e síntese dos resultados obtidos nas pesquisas objetos de estudo, o qual, ao final, entrega o atual consenso sobre o que se conhece de mais atual em uma temática específica e se encontra distribuído em estudos independentes (Souza, Silva \& Carvalho, 2010). A condução do estudo ocorreu com as seguintes etapas: elaboração da questão de pesquisa, busca dos estudos primários, extração de dados, avaliação dos estudos primários, análise e síntese dos resultados e apresentação.

A revisão seguiu as recomendações dos Principais Itens para Relatar Revisões sistemáticas e Meta-análises (PRISMA) (Galvão, Pansani \& Harrad, 2015) e orientou-se a partir da elaboração da pergunta norteadora, fundamentada com base na estratégia/estrutura PICO (P: pacientes/população; I: intervenção; C: comparação/controle; O: resultado/outcome) (LandaRamírez \& Arredondo-Pantaleón, 2014) (Quadro 1): Qual a percepção de alunos e cirurgiões-dentistas acerca dos resíduos gerados após atendimentos clínicos e seu impacto ambiental?

Quadro 1. Estratégia seguindo o acrônimo PICO (População/Intervenção/Comparação e Resultado).

\begin{tabular}{|c|c|l|}
\hline $\begin{array}{c}\text { ACRÔNIM } \\
\text { O }\end{array}$ & DEFINIÇÃo & \multicolumn{1}{c|}{ DESCRIÇÃo } \\
\hline P & População & Cirurgiões-dentistas e acadêmicos de Odontologia \\
\hline I & Intervenção & $\begin{array}{l}\text { Informação dada a respeito do correto gerenciamento dos resíduos gerados em } \\
\text { atendimento e seu impacto ambiental }\end{array}$ \\
\hline C & Comparação & $\begin{array}{l}\text { Cirurgiões-dentistas e/ou acadêmicos de Odontologia que não receberam quaisquer } \\
\text { informação a respeito do correto gerenciamento dos resíduos gerados em atendimento } \\
\text { e seu impacto ambiental }\end{array}$ \\
\hline $\mathbf{O}$ & Resultado & O correto gerenciamento dos materiais odontológicos \\
\hline
\end{tabular}

Fonte: Autores (2021).

Foram realizadas buscas eletrônicas de artigos nas bases de dados PubMed, Scientific Electronic Library Online (SciELO), Medical Literature Analysis and Retrieval System Online (MEDLINE), Literatura Latino-americana e do Caribe em Ciências da Saúde (LILACS) e Bibliografia Brasileira de Odontologia (BBO), sendo estes três últimos acessados através da Biblioteca Virtual de Saúde (BVS). Os mesmos critérios de buscas foram usados em todas as bases de dados, de modo a realizar a captação por meio dos títulos, resumos e assuntos, sendo três estratégias aplicadas às bases em questão, segundo os mecanismos disponíveis nas abas de pesquisas das bases, a fim de aumentar a abrangência dos trabalhos, intercalando com palavras e DeCS/MeSH. Tal distinção nas estratégias se deu em virtude da diferença entre os índices disponíveis nas bases, uma vez que na BVS se encontram as opções utilizáveis "Título, Resumo, Assunto; Título; Resumo", SciELO "Todos os Índices; Título; Resumo" e PubMed "All Fields; Title; Title/Abstract", com as respectivas buscas (Quadro 2). Desta forma, os descritores utilizados para a busca de artigos com base nos Descritores em Ciências da Saúde (DeCS) e Medical Subject Heading (MeSH) foram: "Odontologia", "Gerenciamento de resíduos" e "Resíduos odontológicos"; "Dentistry", "Waste management" e "Dental waste"; enquanto que as palavras Odontologia sustentável e Sustainable dentistry foram combinadas com os descritores supracitados entre as estratégias. No rastreamento das publicações foi utilizado o operador booleano "AND", de forma a combinar as palavras mencionadas acima (Quadro 2).

O processo de seleção dos artigos foi realizado em três etapas: (1) remoção de duplicatas, (2) leitura do título e resumo; e (3) análise de texto na íntegra. O levantamento dos artigos foi realizado nos meses de Fevereiro a Março de 2021, o qual obteve 
sua amostra final contemplando estudos publicados entre os anos de 2008 a 2019. Para a inclusão dos artigos, foram empregados os seguintes critérios: Artigos publicados e indexados nas bases de dados selecionadas, escritos em inglês, português ou espanhol. Foram excluídos artigos do tipo estudos de revisão, dissertações, teses, relatos de caso e artigos não condizentes com a temática.

Quadro 2. Estratégia de busca utilizada nas bases de dados selecionadas.

\begin{tabular}{|c|c|}
\hline Base & Estratégia utilizada \\
\hline PubMed & $\begin{array}{l}\text { “All Fields": ((Dental Waste) AND (dentistry)) AND (sustainable dentistry); } \\
\text { "Title": (dentistry[Title/Abstract]) AND (waste management[Title/Abstract]). } \\
\text { "Title/Abstract": (dentistry[Title/Abstract]) AND (waste management[Title/Abstract]). }\end{array}$ \\
\hline BVS & $\begin{array}{l}\text { "Título, resumo, assunto": (odontologia sustentavel) AND (residuos odontologicos) AND (odontologia); } \\
\text { "Título": (odontologia) AND (gerenciamento de residuos); } \\
\text { "Resumo": (odontologia) AND (gerenciamento de residuos). }\end{array}$ \\
\hline SciELO & $\begin{array}{l}\text { "Todos os índices": (odontologia sustentavel) AND (residuos odontologicos) AND (odontologia); } \\
\text { "Título": (ti:((odontologia) AND (gerenciamento de residuos))); } \\
\text { "Resumo": (ab:((odontologia) AND (gerenciamento de residuos))). }\end{array}$ \\
\hline
\end{tabular}

Fonte: Autores (2021).

Após a seleção dos artigos em cada base de dados, os mesmos foram enviados para um gerenciador de referências e software de processamento (Mendeley® Desktop, Elsevier) para melhor coleta das informações.

\section{Resultados}

Após as buscas feitas seguindo todo o rigor descrito anteriormente, foi obtido um total de 49 artigos. Destes, no momento da triagem, foram removidos 14 em decorrência de duplicidade entre bases e posteriormente removidos mais 4 após avaliação dos títulos, totalizando 18 artigos excluídos e ficando 31 para terem seus resumos avaliados segundo os critérios de elegibilidade. Neste tocante, realizou-se a seleção através da exclusão nos trabalhos inelegíveis, onde foram excluídos 18 e 13 seguiram para avaliação na íntegra. Por fim, destes 13 artigos selecionados para leitura na íntegra, 5 foram excluídos, seja por não contemplarem a pergunta norteadora ou mesmo por ainda haver sido detectada uma tese de dissertação em meio aos lidos na íntegra. A amostra final do presente trabalho foi composta por 8 estudos primários que cumpriram todos os critérios de elegibilidade (Figura 1). 
Figura 1. Fluxograma PRISMA de estudos selecionados.

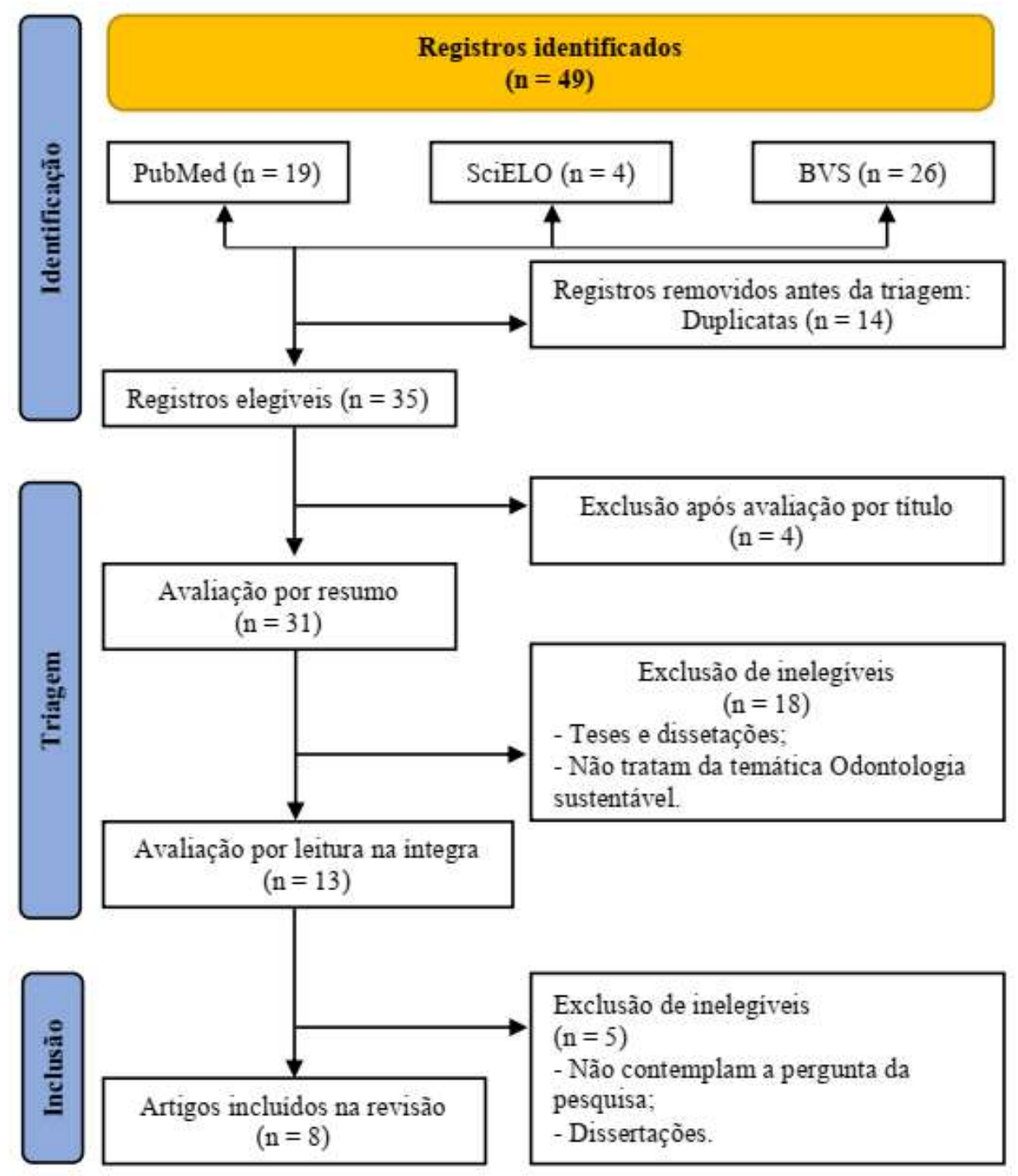

Fonte: Autores. Adaptado PRISMA (2020).

Nesse sentido, as autoras conduziram uma análise independente e cega com base nos critérios de elegibilidade. As divergências de opinião sobre a inclusão ou exclusão de um estudo foram resolvidas por consenso, com o envolvimento de um terceiro revisor, quando necessário.

Todos os estudos apresentaram preocupações em inferir acerca do conhecimento sobre os RSS, seja a amostra do estudo composta por acadêmicos, como visto em Garbin et al. (2015); Fialho et al. (2016) e Silva et al. (2019), composta por profissionais da Odontologia, demonstrado em Hidalgo, Garbin, Rovida e Garbin (2013) e Grose, Richardson, Mills, Moles e Nasser (2016), ou, como podemos perceber em Corrêa, Lunardi e Santos (2008) ambos os casos, acadêmicos e profissionais da Odontologia e/ou como demonstrado em Gomes, Miguel, Rocha e Gomes (2014) que teve como amostra não só acadêmicos, professores e coordenadores de curso, mas também os profissionais terceirizados responsáveis pela coleta do campus. Os principais aspectos metodológicos e resultados observados podem ser vistos no Quadro 3. 
Quadro 3. Características dos estudos incluídos na revisão.

\begin{tabular}{|c|c|c|c|c|}
\hline $\begin{array}{l}\text { Autores/ } \\
\text { Ano }\end{array}$ & Objetivo & Tamanho da amostra & $\begin{array}{l}\text { Delineamento } \\
\text { do estudo }\end{array}$ & Principais desfechos \\
\hline $\begin{array}{l}\text { Corrêa, Lunardi, } \\
\text { Santos et al, } \\
2008 .\end{array}$ & $\begin{array}{l}\text { Conhecer como vem ocorrendo } \\
\text { a construção do saber sobre RSS } \\
\text { no processo de formação dos } \\
\text { acadêmicos dos cursos da área } \\
\text { da saúde: Enfermagem e } \\
\text { Obstetrícia, Odontologia, } \\
\text { Medicina Veterinária e } \\
\text { Medicina. }\end{array}$ & $\begin{array}{l}4 \text { coordenadores de } \\
\text { colegiados dos cursos, } 3 \\
\text { professores e } 14 \text { acadêmicos } \\
\text { de graduação que estavam } \\
\text { cursando o último semestre. }\end{array}$ & $\begin{array}{l}\text { Estudo } \\
\text { qualitativo. }\end{array}$ & $\begin{array}{l}\text { Evidenciou-se a necessidade de } \\
\text { comprometimento dos docentes, } \\
\text { com atitudes de desafio em suas } \\
\text { práticas pedagógicas para } \\
\text { instigar a busca dessas novas } \\
\text { compreensões e a reavaliação da } \\
\text { educação integral sobre os } \\
\text { manejos dos RSS. }\end{array}$ \\
\hline $\begin{array}{l}\text { Hidalgo et al, } \\
2013 \text {. }\end{array}$ & \begin{tabular}{lrrr} 
Verificar o & \multicolumn{2}{c}{ processo } & de \\
gerenciamento & dos & resíduos \\
odontológicos & no & serviço \\
público de saúde & em & 11 \\
municípios (Região Central do & \\
Departamento & & & \\
Regional de & Saúde & II & - \\
Araçatuba-SP). & & &
\end{tabular} & $\begin{array}{l}50 \quad \text { estabelecimentos } \\
\text { públicos de saúde com } \\
\text { atendimentos odontológicos } \\
\text { em } 11 \text { municípios. }\end{array}$ & $\begin{array}{l}\text { Estudo } \\
\text { descritivo, } \\
\text { exploratório e } \\
\text { de caráter } \\
\text { transversal. }\end{array}$ & $\begin{array}{l}\text { Os protocolos adotados pela } \\
\text { rede pública de saúde bucal, em } \\
\text { relação ao gerenciamento de } \\
\text { RSS, apresentaram várias falhas } \\
\text { visto que o plano desse } \\
\text { gerenciamento não é uma } \\
\text { realidade nas unidades } \\
\text { odontológicas públicas e, por } \\
\text { fim, apontou que transformar } \\
\text { essa realidade pressupõe um } \\
\text { trabalho integrado entre todos os } \\
\text { segmentos da sociedade. }\end{array}$ \\
\hline $\begin{array}{l}\text { Gomes et al, } \\
2014 .\end{array}$ & $\begin{array}{l}\text { Avaliar o conhecimento } \\
\text { acadêmicos dos cursos de } \\
\text { Odontologia, Nutrição e } \\
\text { Farmácia do campus III } \\
\text { Jardim Botânico, da } \\
\text { Universidade Federal do Paraná } \\
\text { (UFPR) sobre } \\
\text { Biossegurança e RSS. }\end{array}$ & $\begin{array}{l}\text { Graduandos: Odontologia } \\
(\mathrm{n}=27), \quad \text { Nutrição } \quad(\mathrm{n}=20), \\
\text { Farmácia } \quad(\mathrm{n}=31), \quad \text { Terapia } \\
\text { Ocupacional } \\
(\mathrm{n}=28), \quad \text { Enfermagem } \\
(\mathrm{n}=24) ; \text { coordenadores dos } \\
\text { cursos } \quad(\mathrm{n}=5) ; \quad \text { gestor } \\
\text { ambiental local } \quad(\mathrm{n}=1) ; \\
\text { manipuladores de resíduos } \\
\text { atuantes no campus }(\mathrm{n}=20) .\end{array}$ & $\begin{array}{l}\text { Pesquisa-ação } \\
\text { com aplicação } \\
\text { de instrumento } \\
\text { avaliativo. }\end{array}$ & $\begin{array}{l}\text { A pesquisa evidenciou uma } \\
\text { lacuna no conhecimento e } \\
\text { atitudes em medidas biosseguras } \\
\text { e responsáveis que limitem } \\
\text { qualquer possibilidade de haver } \\
\text { incidente e conclui sugerindo } \\
\text { mais ações educativas neste } \\
\text { sentido. }\end{array}$ \\
\hline Leal, 2015. & $\begin{array}{l}\text { Orientar na formação } \\
\text { responsável e ética do futuro } \\
\text { profissional quanto à saúde } \\
\text { humana e ambiental, com vistas } \\
\text { à saúde pública. }\end{array}$ & NÃO & $\begin{array}{ll}\text { Estudo } & \text { de } \\
\text { revisão } & \text { da } \\
\text { literatura } & \text { de } \\
\text { caráter } & \\
\text { descritivo, } & \\
\text { exploratório } & \text { e } \\
\text { bibliográfico. }\end{array}$ & $\begin{array}{l}\text { O estudo deixou claro a } \\
\text { necessidade da compreensão } \\
\text { clara da relação entre risco, } \\
\text { saúde ocupacional, saúde } \\
\text { ambiental, biossegurança e } \\
\text { gerenciamento de resíduos. } \\
\text { Passando a responsabilidade às } \\
\text { IES em formular esses } \\
\text { conhecimentos e valores nos } \\
\text { futuros profissionais da saúde, } \\
\text { com posturas mais éticas e } \\
\text { conscientes do seu papel na } \\
\text { sociedade. }\end{array}$ \\
\hline $\begin{array}{l}\text { Garbin et al, } \\
2015 .\end{array}$ & $\begin{array}{l}\text { Verificar o grau de } \\
\text { conhecimento dos } \\
\text { de odontologia } \\
\text { gerenciamento de resíduos de } \\
\text { serviços de saúde. }\end{array}$ & $\begin{array}{l}140 \quad \text { graduandos em } \\
\text { atividade clínica em 2012.1. }\end{array}$ & $\begin{array}{l}\text { Estudo } \\
\text { descritivo e de } \\
\text { caráter } \\
\text { transversal. }\end{array}$ & $\begin{array}{l}\text { O conhecimento dos graduandos } \\
\text { sobre o tema ainda é falho, o que } \\
\text { sugere a necessidade de } \\
\text { implementar } \\
\text { interdisciplinares } \\
\text { teórico/práticas e meios de } \\
\text { sensibilizar os futuros } \\
\text { profissionais para tornar-se uma } \\
\text { prática constante. }\end{array}$ \\
\hline
\end{tabular}




\begin{tabular}{|c|c|c|c|c|}
\hline $\begin{array}{l}\text { Grose et al, } \\
2016 \text {. }\end{array}$ & $\begin{array}{l}\text { Explorar o conhecimento e } \\
\text { percepção da } \\
\text { em ustentabilidade } \\
\text { odontológico, em North Devon, } \\
\text { Inglaterra. }\end{array}$ & NÃO DISPONÍVEL & $\begin{array}{l}\text { Estudo } \\
\text { qualitativo. }\end{array}$ & $\begin{array}{l}\text { O estudo fomentou a } \\
\text { necessidade de uma nova } \\
\text { configuração de serviços de } \\
\text { saúde, onde o atendimento } \\
\text { clínico gere o mínimo de danos } \\
\text { ambientais possíveis, } \\
\text { embasando suas estratégias nos } \\
\text { três Rs: reduzir, reutilizar e } \\
\text { reciclar. }\end{array}$ \\
\hline $\begin{array}{l}\text { Fialho et al, } \\
2016\end{array}$ & $\begin{array}{l}\text { Avaliar o efeito de uma } \\
\text { intervenção educacional com } \\
\text { acadêmicos a respeito do } \\
\text { gerenciamento dos RSS e a } \\
\text { prática de condutas sustentáveis. }\end{array}$ & $\begin{array}{l}100 \quad \text { graduandos } \mathrm{em} \\
\text { odontologia da UFMA. }\end{array}$ & $\begin{array}{l}\text { Estudo } \\
\text { Intervencionista }\end{array}$ & $\begin{array}{l}\text { A intervenção educacional no } \\
\text { gerenciamento de resíduos } \\
\text { recicláveis com base na } \\
\text { Odontologia sustentável foi um } \\
\text { mecanismo válido no } \\
\text { aprendizado dos acadêmicos, } \\
\text { sendo capaz de modificar a } \\
\text { prática clínica e a concepção de } \\
\text { uma relação harmônica com o } \\
\text { meio ambiente. }\end{array}$ \\
\hline $\begin{array}{l}\text { Da Silva et al, } \\
2019 .\end{array}$ & $\begin{array}{l}\text { Analisar a percepção clínica, } \\
\text { ética e legal de acadêmicos do } \\
\text { Curso de Odontologia da } \\
\text { Universidade Federal do Piauí } \\
\text { (UFPI) sobre o gerenciamento } \\
\text { dos RSS. }\end{array}$ & $\begin{array}{l}41 \text { acadêmicos do } 8^{\circ}(n=23) \\
\text { e } 9^{\circ}(n=18) \text { períodos. }\end{array}$ & $\begin{array}{l}\text { Estudo } \\
\text { observacional } \\
\text { transversal. }\end{array}$ & $\begin{array}{l}\text { O conhecimento dos estudantes } \\
\text { deste estudo sobre o } \\
\text { gerenciamento de RSS foi } \\
\text { satisfatório quanto aos aspectos } \\
\text { clínicos e éticos, porém } \\
\text { insuficiente quanto aos aspectos } \\
\text { legais. }\end{array}$ \\
\hline
\end{tabular}

Fonte: Autores (2021).

Vale ressaltar, que no estudo apresentado por Leal (2015) não foi possível detectar qual o tamanho da sua amostra, uma vez que o trabalho é de caráter de revisão da literatura, não havendo, portanto, um aspecto quantitativo; não obstante, Grose et al. (2016) apresentam um trabalho qualitativo, entretanto seu trabalho há a aplicação de questionário, porém no decorrer da pesquisa não há a abertura, ao menos, de inferir quantos foram os entrevistados, uma vez que quando citados fala-se apenas de "funcionários da clínica".

Curiosamente, o estudo de Fialho et al. (2016) foi o único achado, dentre os elegíveis nesta revisão, que apresentou uma abordagem intervencionista, onde foi inicialmente aplicado um questionário contendo 10 perguntas sobre o gerenciamento de resíduos recicláveis com base na odontologia sustentável, a partir disso, durante 11 meses, os pesquisadores aplicaram intervenções, por exemplo, palestras, exposição de banners, distribuição de panfletos e cartilhas sobre a temática geral da intervenção "Eu Pratico Odontologia Sustentável”, não obstante, puseram nas clínicas da faculdade onde foi feita a pesquisa, lixeiras plásticas devidamente identificadas para separação do lixo. Posteriormente, aplicaram sobre o mesmo grupo, um outro questionário, as mesmas 10 perguntas iniciais acrescidas mais três (03) que buscavam inferir acerca da opinião sobre o material apresentado nas formas de intervenções. Dentre os principais resultados, foi possível perceber que o conhecimento dos acadêmicos a respeito do correto descarte do lixo odontológico e material perfuro cortante praticamente não hou ve mudança significativa, entretanto, se tratando do descarte de materiais tóxicos como amálgama, revelador e fixador, além das embalagens radiográficas, observou-se o oposto, com percentuais satisfatórios demonstrando aumento no conhecimento, por parte dos acadêmicos, destas indagações.

\section{Discussão}

É necessário entender que o homem é parte integrante da natureza, o que significa que este requer um ambiente saudável 
para sua sobrevivência, e que qualquer dano causado à natureza, reflete em impactos negativos e/ou danos à saúde pública, assim a relação homem/natureza deva sempre ser posta em discussão. Nesse contexto, surge a biossegurança como forma de minimizar ou anular impactos causados pela atividade humana, enquanto gerador de resíduos, sobre a saúde dos envolvidos, seja o próprio profissional, seu(s) auxiliar(es), paciente(s), bem como do meio ambiente (Leal, 2015).

Diante do exposto, a biossegurança é, para Gomes et al. (2014), ações de prevenção de acidentes, que visam a proteção do trabalhador; minimização dos riscos inerentes a diversas atividades; visa a saúde do ser vivo e preservação do meio ambiente, além de manter o gerenciamento correto dos resíduos de serviços de saúde. Neste tocante, uma contaminação por microrganismos, no ambiente odontológico, representa uma ameaça classificada como risco biológico e as práticas odontológicas expõem direta ou indiretamente todos os profissionais e pacientes envolvidos, desde o momento do atendimento até o gerenciamento daqueles resíduos, pondo a vida de todos estes em risco.

Os resíduos de serviços de saúde (RSS) são aqueles gerados em qualquer âmbito de assistência à saúde humana ou animal (Leal, 2015) e dividem-se, segundo a Resolução 357 do CONAMA, Conselho Nacional de Meio Ambiente [CONAMA] (2005), em 5 grupos, sendo estes: A, B, C, D e E, sendo, porém , aplicáveis à Odontologia os seguintes: grupo A, aqueles resíduos que apresentam riscos biológicos; grupo B, os resíduos que continham substâncias químicas; grupo C para resíduos contidos de radionuclídeos e grupo E, resíduos que em seu conteúdo possuam materiais perfurocortantes e/ou escarificantes. Questionados a respeito do conhecimento acerca dos RSS, 87,8\% dos acadêmicos entrevistados no estudo de Silva et al. (2019), demonstraram conhecimento acerca do que seria RSS.

No que tange ao gerenciamento de resíduos sólidos de saúde Garbin et al. (2015), discorrem acerca PGRSS, o qual apresenta ações relativas às etapas do manejo de todo RSS gerado em estabelecimentos propriamente de saúde e que objetiva minimizar a produção e proporcionar aos resíduos gerados um encaminhamento seguro que seriam aspectos referentes à geração, segregação, acondicionamento, tratamento interno, coleta, transporte interno e externo, bem como a destinação final e consequente proteção à saúde pública; conhecimento este acerca do PGRSS, que 70,7\% dos graduandos no estudo de Garbin et al. (2015) afirmaram não possuir, o que contrasta com os achados de Silva et al. (2019), onde 100\% dos seus entrevistados responderam corretamente quando questionados a respeito do recipiente para acondicionamento do material perfurocortante desprezado, forma correta de descarte dos líquidos revelador e fixador de radiografias e sobre o acondicionamento de mercúrio residual (97,6\%), o que mostra que os estudantes apresentaram ter ciência sobre a importância do tratamento de resíduos.

Para sanar tais equívocos no conhecimento dos acadêmicos, Corrêa et al. (2008) afirma que a educação, quando voltada para a totalidade, irá colaborar para uma nova forma de compreender o processo de construção do conhecimento, e os docentes devem se comprometer a trabalhar de forma interdisciplinar, de forma ética, com diálogo, solidariedade, cooperação e cidadania, com o propósito de formar profissionais mais capacitados, e que o espaço acadêmico seja um transformador crítico dessa realidade de degradação ambiental, que afeta a sociedade em sua totalidade. Outrossim, Fialho et al. (2016) corrobora a ideia supracitada e afirma que a intervenção educacional no gerenciamento de resíduos é fundamental para a mudança na realidade da Odontologia, e a prática clínica pode ser conduzida a uma relação mais harmônica com o meio ambiente, assim, os acadêmicos seriam os precursores dessa nova realidade.

Em adição aos fatos elencados, o estudo de Hidalgo et al. (2013) sai da esfera acadêmica e apresenta outra realidade, a do serviço em saúde pública, onde teoricamente os resultados deveriam ser mais satisfatórios, pois, há um controle maior sobre esses locais por parte do Estado, entretanto, na população analisada, o gerenciamento de resíduos de saúde apresentam falhas e ainda não é uma realidade nas unidades odontológicas públicas, isso demonstra a vulnerabilidade que se encontra o serviço público e demanda atenção e responsabilização dos órgãos públicos gerenciadores. Em paralelo, o escrito de Grose et al. (2016) apresenta uma realidade diferente, a esfera privada, entretanto, o mesmo é evidenciado, um despreparo por parte dos profissionais cirurgiões- dentistas quando se refere a Odontologia Sustentável. Por outro lado, reduzir, reciclar e reutilizar ainda são os pilares 
mais fortes de uma prática odontológica mais saudável para o meio ambiente.

\section{Conclusão}

A literatura consultada apontou que a educação ambiental relacionada com a prática clínica odontológica ainda não é uma realidade vivenciada cotidianamente nos cursos de Odontologia, como também, as atitudes sustentáveis não são praticadas por cirurgiões-dentistas já formados. Apesar de alguns resultados serem positivos quanto ao conhecimento sobre os RSS, as atitudes praticadas de sustentabilidade não são realizadas. Dessa forma, é evidente a necessidade de mais ações intervencionistas em saúde, tanto por parte dos gestores com a efetiva prática do PGRSS nos estabelecimentos de saúde públicos, quanto por cirurgiões-dentistas em seus consultórios particulares. Ademais, sugerimos maior desenvolvimento de pesquisas na área por se tratar de um tema relevante, atual e que gera impacto, uma vez que a evidência científica é o que dá subsídio para os argumentos que, de fato, causam mudança na sociedade. Não obstante, o incentivo nas universidades à prática de uma Odontologia sustentável é fundamental, para formar profissionais capacitados, eticamente politizados e socialmente aptos a realizar uma conduta consciente com a vida e o meio ambiente. A formação dos futuros cirurgiões-dentistas atentos à sustentabilidade da Odontologia deve se apoiar nas mudanças das matrizes curriculares e o estímulo por parte de docentes e coordenadores, desta forma, proporcionará mudança positiva no impacto ambiental da prática profissional.

\section{Referências}

Associação Brasileira de Normas Técnicas. NBR 12.808: resíduos de serviços de saúde: classificação. ABNT. São Paulo.

Brasil. Conselho Nacional de Meio Ambiente. Resolução CONAMA n. 357, de (2005, mar 16). Serviços odontológicos: prevenção e controle de riscos. www.mma.gov.br/port/conama/res/res05/res35705.pdf.

Brasil. Ministério da Saúde. Agência Nacional de Vigilância Sanitária. (2006). Serviços Odontológicos: prevenção e controle de riscos. ANVISA.

Corrêa, L. B., Lunardi, V. L., \& Santos, S. S. C. (2008). Construção do saber sobre resíduos sólidos de serviços de saúde na formação em saúde.

Diaz, P. S., Soares, S. G. A., Camponogara, S., Saldanha, V. S., Menegat, R. P., \& Rossato, G. C. (2013). Waste Management: a descriptive-exploratory study in the emergency room of a teaching hospital. Online Brazilian Journal of Nursing, 12(4), 964-974.

Fialho, L. M., Poluha, R. L., Soares, B. L. L., Sousa, B. M. D., Freitas-Anceles, J. D. F. D. S. D., Melo Neto, C. L. D. M., ... \& Silva, V. C. D. (2016). Intervenção educacional no gerenciamento de resíduos com base na odontologia sustentável. Rev. Odontol. Araçatuba (Online), 41-45.

Galvão, T. F., Pansani, T. D. S. A., \& Harrad, D. (2015). Principais itens para relatar Revisões sistemáticas e Meta-análises: A recomendação PRISMA. Epidemiologia e Serviços de Saúde, 24, 335-342.

Garbin, A. J. Í., Gomes, A. M. P., Souza, M. P., Arcieri, R. M., Rovida, T. A. S., \& Garbin, C. A. S. (2015). A responsabilidade socioambiental na formação acadêmica. O Mundo da Saúde, 39(1), 119-125.

Garcia, L. P., \& Zanetti-Ramos, B. G. (2004). Gerenciamento dos resíduos de serviços de saúde: uma questão de biossegurança. Cadernos de Saúde Pública, 20, 744-752.

Gessner, R., Piosiadlo, L. C. M., Fonseca, R. M. G. S., \& Larocca, L. M. (2013). O manejo dos resíduos dos serviços de saúde: um problema a ser enfrentado. Cogitare Enfermagem, 18(1).

Gomes, L. C., Miguel, Y. D., Rocha, T. C., \& Gomes, E. C. (2014). Biossegurança e resíduos de serviços de saúde no cotidiano acadêmico. Revista de Ciências Farmacêuticas Básica e Aplicada, 35(3).

Gomes, L. P., \& Esteves, R. V. R. (2012). Análise do sistema de gerenciamento dos resíduos de serviços de saúde nos municípios da bacia hidrográfica do Rio dos Sinos, Rio Grande do Sul, Brasil. Engenharia Sanitária e Ambiental, 17, 377-384.

Grose, J., Richardson, J., Mills, I., Moles, D., \& Nasser, M. (2016). Exploring attitudes and knowledge of climate change and sustainability in a dental practice: A feasibility study into resource management. British dental journal, 220(4), 187-191.

Hidalgo, L. R. D. C., Garbin, A. J. I., Rovida, T. A. S., \& Garbin, C. A. S. (2013). Gerenciamento de resíduos odontológicos no serviço público. Revista de Odontologia da UNESP, 42, 243-250.

Kemparaj, V. M., Panchmal, G. S., \& Kadalur, U. G. (2018). The Top 10 ethical challenges in dental practice in Indian scenario: Dentist perspective. Contemporary clinical dentistry, 9(1), 97.

Landa-Ramírez, E., \& de Jesús Arredondo-Pantaleón, A. (2014). Herramienta PICO para la formulación y búsqueda de preguntas clínicamente relevantes en la psicooncología basada en la evidencia. Psicooncología, 11. 
Research, Society and Development, v.10, n.16, e451101624218, 2021

(CC BY 4.0) | ISSN 2525-3409 | DOI: http://dx.doi.org/10.33448/rsd-v10i16.24218

Leal, C. A. G. (2015). Biossegurança e gerenciamento de resíduos de serviços de saúde: a importância na formação do profissional da Odontologia na perspectiva da saúde humana e ambiental. Revista da ABENO, 15(2), 82-94.

Molina, A. B., Bueno, C. S., Aida, C. A., Castanheira, G. M., Hada, R. A., Ishikiriyama, Y. T., ... \& Brassaroto, G. (2017). A radiologia odontológica e o meio ambiente. Revista de Odontologia da Universidade Cidade de São Paulo, 26(1), 61-70.

Pinto, M. L., Motta, L. J. \& Raptopoulos, G. S. (2015). Ações sustentáveis e gestão de resíduos em Odontologia. III Simpósio Internacional de Gestão de Projetos e II Simpósio Internacional de Inovação e Sustentabilidade.

Silva, D. P., Sousa, M. L., Sipaúba, G. M. O., Moura, M. S. L., Silva Valente, V., \& Moura, C. D. V. S. (2019). Percepção clínica, ética e legal de acadêmicos de Odontologia sobre gerenciamento de resíduos de serviços de saúde. Revista da ABENO, 19(3), 113-122.

Souza, M. T. D., Silva, M. D. D., \& Carvalho, R. D. (2010). Revisão integrativa: o que é e como fazer. Einstein (São Paulo), 8, 102-106.

Turma do Bem. (2009). Manual do Dentista Verde. e-Book, p. 40. https://turmadobem.org.br/br/manual_dentista_verde.pdf. 